\title{
Burn injury mortality in patients with preexisting and new onset renal disease
}

\author{
Laquanda T. Knowlin, Laura Purcell, Bruce A. Cairns, Anthony G. Charles* \\ Department of Surgery, University of North Carolina at Chapel Hill, North Carolina Jaycee Burn Center, USA
}

\section{A R T I C L E I N F O}

Article history:

Received 2 August 2017

Accepted 27 February 2018

Keywords:

Burn injury

Renal disease

Acute kidney injury

Chronic renal disease

Burn mortality
A B S T R A C T

Introduction: We sought to examine the impact of preexisting and new onset renal disease on burn injury mortality.

Methods: Retrospective analysis of patients admitted to a regional burn center from 2002-2012 was performed. Variables analyzed included demographics, burn mechanism, inhalation injury status, and \% TBSA. Poisson regression was performed to estimate risk of in-hospital burn mortality.

Results: There were a total of 7640 patients over the study period. The adjusted 60-day risk of in-hospital mortality in patients with preexisting renal disease (PRD was 3 times higher compared to patients with no preexisting renal disease ( $\mathrm{IRR}=3.22,95 \% \mathrm{CI}=1.26-8.25$ ). The adjusted 60 -day risk of mortality is 2 times higher for patients with new onset renal disease compared to those without (IRR $=2.11,95 \%$ $\mathrm{CI}=1.55-2.87$ )

Conclusion: Preexisting and new onset renal disease results in a significantly higher risk of mortality following burn injury compared to patients without renal disease. Prevention of new onset renal injury and careful management of patients with preexisting renal disease to prevent exacerbation should be pursued.

\section{Introduction}

In the United States, 23 to 30 million adults have chronic kidney disease (CKD) with millions of others at increased risk. ${ }^{1,2}$ Chronic kidney disease is defined as any condition that causes reduced renal function over a long period of time. ${ }^{3}$ Diagnosis is based on the estimated glomerular filtration rate (GFR), the rate at which kidney filter waste, and proteinuria, the amount of protein present in the urine. There are five stages of CKD ranging from kidney damage with normal function to end-stage renal disease (ESRD) requiring dialysis or transplantation. The two main risk factors for CKD are diabetes mellitus and hypertension. Individuals with preexisting CKD and concomitant diabetes or cardiovascular disease have increased risk of mortality. ${ }^{3}$ Specifically, adults with CKD have higher incidence of premature death from cardiovascular disease compared to matched cohorts without CKD. ${ }^{2,4}$

Acute renal failure (ARF), characterized by sudden loss of the ability of the kidneys to excrete waste, concentrate urine, conserve

\footnotetext{
* Corresponding author. UNC School of Medicine, University of North Carolina, 4008 Burnett Womack Building, CB 7228, USA

E-mail address: anthchar@med.unc.edu (A.G. Charles).
}

electrolytes, and maintain fluid homeostasis, is a frequent clinical problem. In the critical care setting it is associated with a mortality of between $50 \%$ and $80 \%{ }^{5}$ The etiopathogenesis of acute kidney injury varies depending on preexisting medical conditions, hemodynamic changes, and the presence of sepsis. ${ }^{6}$ ARF is one of the major burn complications and is accompanied by high mortality. In burns, most ARF occurs either immediately after injury or with delayed presentation when sepsis develops.

Previously published studies have investigated the effects of ARF on burn injury mortality but no other study has examined the impact of preexisting renal disease on burn injury mortality. We therefore sought to examine the impact of both preexisting and new onset renal disease on burn injury outcomes. We hypothesize that pre-existing and new onset renal disease will result in increased in-hospital mortality following burn injury compared to patients with the absence of renal disease.

\section{Methods}

This is a retrospective study of all burn patients admitted to the North Carolina Jaycee Burn Center from 2002 to 2012. The North Carolina Jaycee Burn Center at the University of North Carolina is an 
American Burn Association verified pediatric and adult center. The center, established in 1981, is a 36-bed single unit facility, which averages more than 1200 acute admissions per year.

The medical records of subjects identified by the UNC Burn database query were reviewed to verify baseline demographic data, injury characteristics, and provide medical comorbidities and complications information. For this study, patients $<18$ years were excluded. In addition, patients with preexisting renal disease were excluded in the secondary analysis for effects of new onset renal disease. Injury characteristics of interest included burn mechanism, \%TBSA burn, presence of inhalation injury, and ventilator status on admission to the burn center. Inhalation injury diagnosis was based on history, physical examination, and/or bronchoscopic examination. All patients in the ICU were treated with the same standard of care (i.e. ventilator management, fluid resuscitation, dialysis use, etc.) at a large academic burn center over the time period of the study. Pre-existing comorbidities were obtained from medical records, as well as history reported by the patient, family, or friends privy to patient's history upon admission to the burn center. The exact cause of death for patients in this cohort is not recorded in the burn registry.

To examine the effect of baseline medical comorbidities on outcome, a Charlson Comorbidity Index (CCI) score was calculated for each patient. The standardized Charlson Index has been reported to accurately predict the probability of mortality within one year for a number of medical conditions. ${ }^{7,8}$ The score is the weighted sum of 17 comorbid conditions, each of which is assigned a weight from 1 to 6 points. ${ }^{9}$ The $\mathrm{CCI}$ was modified for this study to exclude renal disease in order to elicit the independent effect of renal disease on burn outcome. Pre-existing comorbidities were identified and recorded based on ICD-9 codes.

The primary outcome of interest in this study was in-hospital mortality. Baseline patient and injury characteristics were compared between groups (preexisting renal disease (PRD) versus no preexisting renal disease (NPRD) and new onset renal disease (NORD) versus no renal disease (NK)) using Analysis of Variance for continuous variables and chi-squared for discrete variables. A twosided $\mathrm{p}$-value $<0.05$ was considered statistically significant. We employed both univariate and multivariate models to determine the relative influence of renal disease on mortality after controlling for covariates. To estimate the incidence risk ratios for mortality, we used a multivariate Poisson regression model controlling for pertinent confounders (age, \%TBSA, mechanism of injury, presence of inhalation injury, other comorbidities using $\mathrm{CCI}$, race, and sex). Age and \%TBSA were modeled as multi-level categorical variables. An interaction term was created to reduce the impact of collinearity between diabetes and renal disease. Sepsis was excluded in the regression model because it was determined to be on the causal pathway that leads to renal failure using a directed acyclic graph (DAG). ${ }^{9}$

Stata/MP (Version 12) (Stata Corp, College Station, TX) was used for all data management and statistical analysis. The University of North Carolina Institutional Review Board approved this study.

\section{Results}

\subsection{The effect of preexisting renal disease}

A total of 5330 patients $\geq 18$ years old were admitted to during the study period and included in analysis for preexisting renal disease effects on burn injury mortality. Preexisting renal disease (PRD) was present in 102 (2\%) patients, which included chronic renal failure $(n=56,55 \%)$, end stage renal disease $(n=15,15 \%)$, and chronic glomerulonephritis $(n=30,29 \%)$. Of those with PRD, $15 \%$ were dialysis dependent. Patients with PRD were more likely to be older (median age 61 vs $41, \mathrm{p}<0.001$ ) and black (57\% vs $25 \%$,

Table 1

Patient demographics and burn characteristics of adult patients, stratified by renal disease status.

\begin{tabular}{|c|c|c|c|}
\hline & Preexisting Renal Disease 102 (1.9\%) & No renal disease 5228 (98.1\%) & p-value ${ }^{a}$ \\
\hline Hospitalization year, n (\%) & & & 0.006 \\
\hline $2002-2005$ & $16(15.7)$ & $1410(29.3)$ & \\
\hline 2006-2009 & $37(36.3)$ & $1726(36.0)$ & \\
\hline 2010-2012 & $49(48.0)$ & $2092(34.7)$ & \\
\hline Sex, n (\%) & & & 0.794 \\
\hline Male & $75(73.5)$ & $3783(72.4)$ & \\
\hline Female & $27(26.5)$ & $1445(27.6)$ & \\
\hline Race/ethnicity, n (\%) & & & $<\mathbf{0 . 0 0 1}$ \\
\hline White & $37(36.3)$ & $2957(56.6)$ & \\
\hline Black & $58(56.9)$ & $1326(25.4)$ & \\
\hline Hispanic & $2(1.9)$ & $365(7.0)$ & \\
\hline Other & $5(4.9)$ & $577(11.0)$ & \\
\hline Age, in years, median (IQR) & $61(50-70)$ & $41(29-54)$ & $<\mathbf{0 . 0 0 1}$ \\
\hline mCCI, median (IQR) & $3(2-3)$ & $0(0-0)$ & $<\mathbf{0 . 0 0 1}$ \\
\hline \multicolumn{4}{|l|}{ Comorbidities, n (\%) } \\
\hline Diabetes & $63(61.8)$ & $534(10.2)$ & $<\mathbf{0 . 0 0 1}$ \\
\hline Prior myocardial infarction & $11(10.8)$ & $117(2.2)$ & $<\mathbf{0 . 0 0 1}$ \\
\hline Burn mechanism, n (\%) & & & 0.129 \\
\hline Flame & $48(47.5)$ & $2887(55.6)$ & \\
\hline Scald & $48(47.5)$ & $1958(37.7)$ & \\
\hline Other burn & $5(5.0)$ & $344(6.6)$ & \\
\hline Unknown & 11 & 39 & - \\
\hline TBSA, median (IQR) & $5(2-12)$ & $5(2-11)$ & 0.795 \\
\hline Inhalation injury, n (\%) & $13(12.7)$ & $509(9.7 \%)$ & 0.314 \\
\hline Dialysis requirement, $\mathrm{n}(\%)$ & $15(14.7)$ & $1(<1)$ & $<\mathbf{0 . 0 0 1}$ \\
\hline Sepsis & $19(18.6)$ & $146(2.8)$ & $<\mathbf{0 . 0 0 1}$ \\
\hline Crude in-hospital mortality & $18(17.6)$ & $262(5.0)$ & $<\mathbf{0 . 0 0 1}$ \\
\hline Hospital LOS, median (IQR) & $23.6(9.0-43.4)$ & $7.0(2.0-14.6)$ & $<\mathbf{0 . 0 0 1}$ \\
\hline
\end{tabular}


Table 2

Crude and adjusted effect of preexisting renal disease on inpatient mortality.

\begin{tabular}{llll}
\hline & HR & $95 \% \mathrm{Cl}^{\mathrm{a}}$ & p-value \\
\hline Crude (overall) & 3.49 & $2.26,5.39$ & $<0.0001$ \\
30 day & 3.31 & $1.71,6.41$ & $<0.001$ \\
60 day & 2.99 & $1.70,5.27$ & $<0.001$ \\
Adjusted $^{\mathrm{b}}$ (overall) & 1.93 & $0.93,4.03$ & 0.076 \\
30 day & 3.71 & $1.47,9.38$ & 0.006 \\
60 day & 3.22 & $1.26,8.25$ & 0.015 \\
\hline
\end{tabular}

Abbreviations: IRR, incidence rate ratio; $\mathrm{CI}$, confidence interval.

a Estimated using a robust variance sandwich estimator.

b Multivariable regression controlling for sex, age, race/ethnicity, non-renal comorbidities using Charlson Comorbidity Index, burn mechanism, total burn surface area (TBSA), dialysis use and inhalational injury; An interaction term was created to reduce the impact of collinearity between preexisting diabetes and renal disease. Age and TBSA were modeled as multi-level categorical variables.

$\mathrm{p}<0.001$ ) compared to patient with NPRD (Table 1 ). There was no significant difference between the two groups in \%TBSA, sex, mechanism of injury, or presence of inhalation injury. Patients with PRD had a higher Charlson Comorbidity Index (2.4 vs. 0.4, $\mathrm{p}<0.001)$ and were more likely to have a history of diabetes mellitus (62\% vs. $10 \%, \mathrm{p}<0.001)$ and/or prior myocardial infarction $(11 \%$ vs $2 \%, \mathrm{p}<0.001$ ). Approximately $15 \%$ (vs. $2 \%$ for NPRD) of patients with PRD had cardiac complications and 19\% (vs 2.8\% for NPRD) developed sepsis $(\mathrm{p}<0.001)$. Patients with PRD had statistically significant longer hospital length of stay with a median 23.6 days (IQR 9-43.4) compared to NPRD patients with a median of 7 days (IQR 2.0-14.6, $\mathrm{p}<0.001$ ).

The crude mortality rate was higher for patients with PRD compared to NPRD ( $18 \%$ vs. $5 \%, \mathrm{p}<0.001$ ). A multivariable Poisson regression model was utilized to determine the incidence risk of burn injury mortality in the PRD vs. NPRD subgroups after adjusting for significant covariates. The adjusted incidence risk ratios (IRR) for in-hospital mortality were significantly higher at 30 and 60 days for patients with PRD versus NPRD patients at $3.71(95 \%$ $\mathrm{CI}=1.47-9.38)$ and $3.22(95 \% \quad \mathrm{CI}=1.26-8.25)$, respectively (Table 2). The cumulative risk of in-hospital mortality was higher for patients with preexisting renal disease within the 60 days of admission compared to healthy renal patients, with or without inhalation injury (Fig. 1). Although the overall adjusted mortality incidence for the PRD cohort was almost 2 times higher $(I R R=1.93$ (NPRD reference group), 95\% confidence interval: 0.93-4.03), this

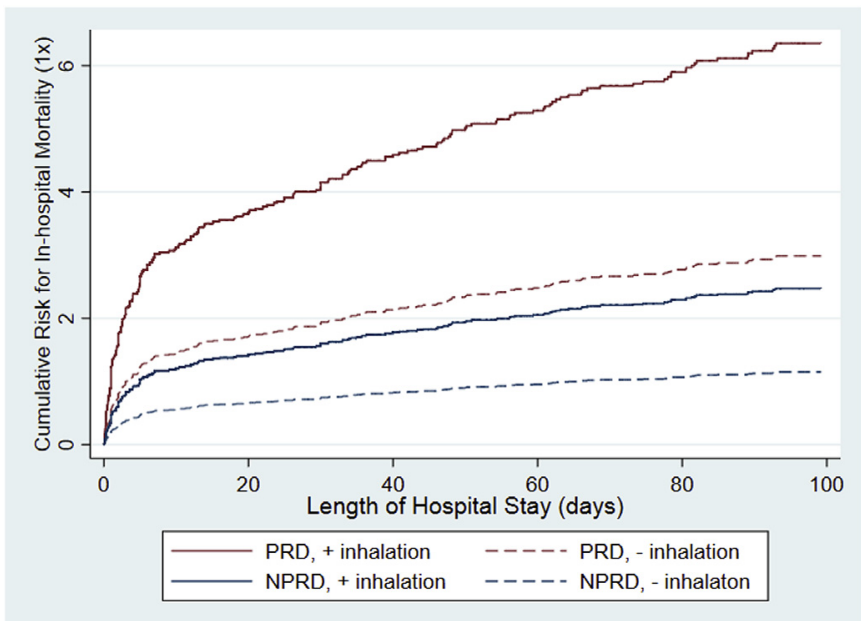

Fig. 1. The cumulative risk of in-hospital mortality for patients with preexisting renal disease within the 60 days of admission compared to healthy renal patients, with or without inhalation injury. was not statistically significant.

\subsection{New onset renal disease}

After exclusion of patients with preexisting renal disease $(\mathrm{n}=102)$, a total of 7539 patients were analyzed to determine the effect of new onset renal disease on burn injury mortality. Of this cohort 194 (2.6\%) developed new onset renal disease, which included acute renal failure ( $\mathrm{n}=189,97 \%)$, acute tubular necrosis $(\mathrm{n}=74,38 \%)$, and acute glomerulonephritis $(\mathrm{n}=6,3 \%)$. In a bivariate analysis, patients with NORD were more likely to be older (median age 53 vs $30, \mathrm{p}<0.001$ ), black ( $41 \%$ vs $27 \%, \mathrm{p}<0.001$ ). In addition, the NORD cohort also had a higher \%TBSA (median 25 (IQR $9-45) \%$ vs. 5 (IQR $2-10) \%, p<0.001$ ) and was more likely to suffer a flame injury ( $75 \%$ vs $45 \%, \mathrm{p}<0.001)$ compared to patient who developed no renal disease (NK) (Table 3). In terms of other medical comorbidities, patients with NORD had a higher Charlson Comorbidity Index ( 1.1 vs $0.3, \mathrm{p}<0.001)$ and were more likely to have a history of diabetes mellitus (18\% vs $7 \%, \mathrm{p}<0.001)$ or prior myocardial infarction ( $9 \%$ vs $1 \%, \mathrm{p}<0.001$ ) compared to the NK cohort. Approximately, $40 \%$ (vs $1.1 \%$ of NK) of patients with NORD developed sepsis $(\mathrm{p}<0.001)$. Patients with NORD had statistically significant longer hospital length of stay (median 45 days (IQR 19-82)) vs. 5 days (IQR 1.6-11.8), p<0.001)) compared to NK cohort.

The crude in-hospital mortality rate was higher for patients with NORD ( $37 \%$ vs $3 \%, \mathrm{p}<0.001$ ). After adjustment for other covariates utilizing a multivariable Poisson regression, the incidence risk of inhospital mortality at 30 and 60 days for the NORD versus NK cohorts were $2.66(\mathrm{CI}=1.75-4.05)$ and $2.11 \quad(\mathrm{CI}=1.55-2.87)$ (Table 4). The cumulative risk of in-hospital mortality was higher for patients who developed NORD within the 60 days of admission compared to patients with no renal issues, particularly in the presence of inhalation injury (Fig. 2). Also, the overall NORD adjusted mortality incidence was almost 2.3 times higher $(\mathrm{IRR}=2.34 \quad$ (NK reference group), 95\% confidence interval: 1.77-3.04).

\section{Discussion}

In this study, we have shown burn patients with preexisting and new onset renal disease have a significantly higher incidence of inhospital mortality compared to burn patients with a history of no renal disease.

There have been no previous studies that have examined the effects of preexisting renal disease on burn injury outcomes. However, multiple studies reveal that AKI is prevalent and associated with increased burn mortality. A systemic review and metaanalysis of published burn articles between 1960 and 2009, revealed a 3-to-6-fold increase in mortality for AKI patients. ${ }^{10}$ When defined based on the Risk, Injury, Failure, Loss and Endstage kidney disease (RIFLE) criteria, $25 \%$ of patients with severe burn injury develop AKI with a median mortality of $34.9 \%$. For patients who required renal replacement therapy, the median mortality was almost $80 \% .{ }^{12}$ In our burn cohort, the crude mortality for patients who developed new onset renal disease was $37 \%$. The cause of AKI may be the determinant factor in burn injury survival.

A previous study on sepsis found a direct relationship between the patient response to treatment and the occurrence of AKI. ${ }^{11}$ In patients with confirmed positive blood cultures, AKI occurs in about $19 \%$ patients with moderate sepsis, $23 \%$ with severe sepsis, and $51 \%$ with septic shock. ${ }^{12}$ Sepsis is the leading cause of death in burn patients and is concomitant in $87 \%$ of burn patients with AKI. ${ }^{13,14}$

Renal failure has been shown to occur during two periods after 
Table 3

Patient demographics and burn characteristics of adult patients, stratified by renal complication status.

\begin{tabular}{|c|c|c|c|}
\hline & New onset renal disease 194 (1.9\%) & No renal disease 7345 (98.1\%) & p-value ${ }^{a}$ \\
\hline Hospitalization year, $\mathbf{n}(\%)$ & & & $<\mathbf{0 . 0 0 1}$ \\
\hline $2002-2005$ & $55(28.3)$ & $1885(25.7)$ & \\
\hline 2006-2009 & $70(36.1)$ & $2486(33.8)$ & \\
\hline $2010-2012$ & $69(35.6)$ & $2974(40.5)$ & \\
\hline Sex, n (\%) & & & 0.224 \\
\hline Male & $141(72.7)$ & $5037(68.6)$ & \\
\hline Female & $53(27.3)$ & $2308(31.4)$ & \\
\hline Race/ethnicity, n (\%) & & & $<\mathbf{0 . 0 0 1}$ \\
\hline White & $87(44.8)$ & $3772(51.4)$ & \\
\hline Black & $79(40.7)$ & $1994(27.2)$ & \\
\hline Hispanic & $8(4.1)$ & $652(8.8)$ & \\
\hline Other & $20(10.3)$ & $924(12.6)$ & \\
\hline Age, in years, median (IQR) & $53(37-64)$ & $30(11-47)$ & $<\mathbf{0 . 0 0 1}$ \\
\hline mCCI, median (IQR) & $3(2-3)$ & $0(0-0)$ & $<\mathbf{0 . 0 0 1}$ \\
\hline \multicolumn{4}{|l|}{ Comorbidities, n (\%) } \\
\hline Diabetes & $34(17.5)$ & $502(6.8)$ & $<\mathbf{0 . 0 0 1}$ \\
\hline Prior myocardial infarction & $18(9.3)$ & $101(1.4)$ & $<\mathbf{0 . 0 0 1}$ \\
\hline Burn mechanism, n (\%) & & & $<\mathbf{0 . 0 0 1}$ \\
\hline Flame & $145(75.5)$ & $3260(44.7)$ & \\
\hline Scald & $35(18.2)$ & $3604(49.1)$ & \\
\hline Other burn & $12(6.3)$ & $434(5.9)$ & \\
\hline Unknown & 2 & 47 & - \\
\hline TBSA, median (IQR) & $25(9-45)$ & $5(2-10)$ & $<\mathbf{0 . 0 0 1}$ \\
\hline Inhalation injury, n (\%) & $72(37.3)$ & $494(6.7 \%)$ & $<\mathbf{0 . 0 0 1}$ \\
\hline Dialysis requirement, n(\%) & $1(<1)$ & $0(0)$ & $<\mathbf{0 . 0 0 1}$ \\
\hline Sepsis & $78(40)$ & $79(1.1)$ & $<\mathbf{0 . 0 0 1}$ \\
\hline Crude in-hospital mortality & $72(37.0)$ & $199(2.7)$ & $<\mathbf{0 . 0 0 1}$ \\
\hline Hospital LOS, median (IQR) & $45.4(19.0-82.0)$ & $5.0(1.6-11.8)$ & $<\mathbf{0 . 0 0 1}$ \\
\hline
\end{tabular}

Abbreviations: IQR, interquartile range; mCCI, Charlson Comorbidity Index excluding renal disease; TBSA, total burn surface area; LOS, length of hospital stay.

a Chi-square and ANOVA tests were used to calculate overall $\mathrm{p}$-values; $\mathrm{p}<0.05$ are in bold.

burn injury. The first period is immediately due to burn shock, a unique combination of distributive and hypovolemic shock manifested by intravascular volume depletion, low pulmonary artery occlusion pressures, elevated systemic vascular resistance, and depressed cardiac output. ${ }^{15,16}$ The second period has a delayed presentation and is usually due to gram-negative sepsis. The pathophysiology of gram-negative sepsis is initiated when the endotoxin lipopolysaccharide (LPS) induces proinflammatory cytokines such as tumor necrosis factor (TNF) that binds to receptors on the glomerular endothelial cell and renal tubular epithelial cells. ${ }^{17,18}$ Nitric oxide (NO) synthase is induced after a chain of reaction resulting in the formation of NO. In large quantities, NO is responsible for the systemic vasodilation seen during sepsis and as a result, leads to septic shock. ${ }^{19}$ The renal system tries to compensate for the volume depletion with vasoconstriction and water retention, which results in a decrease in GFR. ${ }^{20}$ It is common to associate hemodynamic factors such as hypotension with AKI seen in severe sepsis patients.

Table 4

Crude and adjusted effect of new onset renal disease on inpatient mortality.

\begin{tabular}{llll}
\hline & $\mathrm{HR}$ & $95 \% \mathrm{Cl}^{\mathrm{a}}$ & p-value \\
\hline Crude (overall) & 13.57 & $10.80,17.06$ & $<0.0001$ \\
30 day & 14.86 & $11.28,19.57$ & $<0.001$ \\
60 day & 15.78 & $11.30,22.03$ & $<0.001$ \\
Adjusted $^{\mathrm{b}}$ (overall) & 2.31 & $1.77,3.04$ & $<0.001$ \\
30 day & 2.66 & $1.75,4.05$ & $<0.001$ \\
60 day & 2.11 & $1.55,2.87$ & $<0.001$ \\
\hline
\end{tabular}

Abbreviations: IRR, incidence rate ratio; $\mathrm{CI}$, confidence interval.

a Estimated using a robust variance sandwich estimator.

b Multivariable regression controlling for sex, age, race/ethnicity, non-renal comorbidities using Charlson Comorbidity Index, burn mechanism, total burn surface area (TBSA), and inhalational injury; An interaction term was created to reduce the impact of collinearity between diabetes and renal disease. Age and TBSA were modeled as multi-level categorical variables.
The ability to predict acute renal failure is still challenging. In a multivariable regression, both burn size and septicemia were the only significant predictors of acute renal failure. ${ }^{21}$ The initial indication of altered renal function in the clinical setting for either acute on chronic or new acute renal failure is oliguria. In the early phase of burn care, it is imperative that hypovolemia is treated with balanced fluid resuscitation ensuring the attainment of an adequate cardiac preload. ${ }^{22}$ The use of colloid fluids in combination with crystalloids as part of a goal direct resuscitation strategy may be helpful. In addition, the use of heart rate, blood pressure, and urine output, as well as diagnostic adjuncts such as echocardiography to visualize the inferior vena cava and assess cardiac contractility and

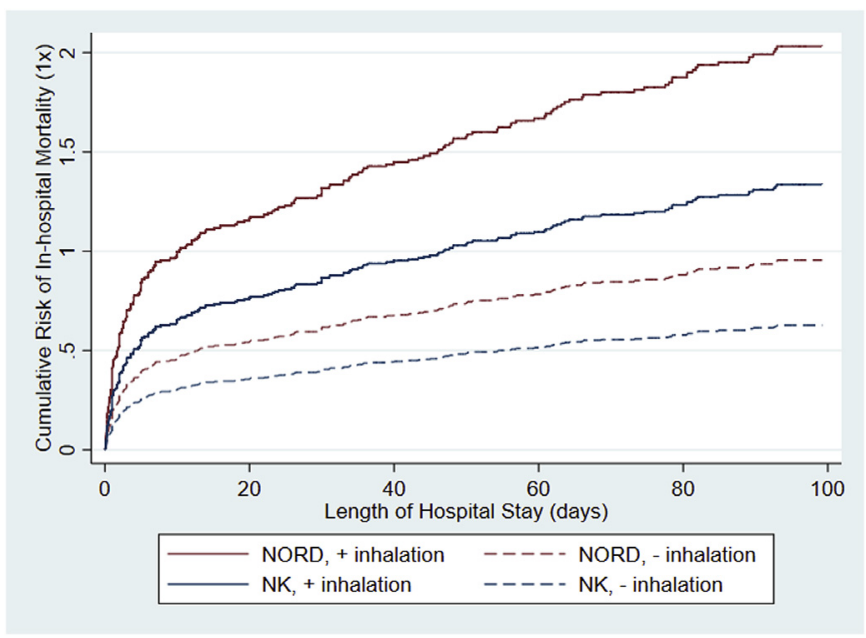

Fig. 2. The cumulative risk of in-hospital mortality for patients who developed New onset Renal disease (NORD) within the 60 days of admission compared to patients with no renal issues (NK), with or without inhalation injury. 
wall motion abnormalities, can help dictate the need for vasopressors.

In comparison, acute kidney injury that is sequelae of sepsis is part of multiple organ dysfunction. Standard protocol of sepsis treatments such as early goal directed therapy, obtaining cultures, source control, administration of broad-spectrum antibiotics, and glycemic control should be initiated. Secondary nephrotoxic injury due to decrease renal perfusion or the use of nephrotoxic medications such as aminoglycoside and contrast agents must be prevented. Utilizing renal dosing of medications to prevent new onset renal disease or acute on chronic renal failure is essential. The regular monitoring of renal function using serum urea and creatinine trends will help indicate renal recovery or need for dialysis. In patients meeting the criteria for renal failure, renal replacement therapy should be initiated as indicated. With advancements in burn care management, there has been a reduction in the number of patient with renal failure requiring RRT with a current incidence between 1 and 3\%. ${ }^{12,16}$

The limitations of this study are those inherent to any study with a retrospective methodology. The pre-existing comorbidities were abstracted from documented diagnosed based on ICD-9 codes and hence subject to coding accuracy. Laboratory values such as blood urea nitrogen, serum creatinine, and GFR were not available in the registry to confirm the diagnosis and to calculate functional excretion of sodium or urea to further delineate the characteristics of the kidney injury. Only inpatient mortality was captured and analyzed, which represents only a portion of all death caused by burn injury. The contribution of frailty and its effect on outcome was not evaluated in our analysis. Finally, this study only includes patients from a single center study; however, the size of our burn cohort studied strengthens our findings.

\section{Conclusion}

Preexisting and new onset renal disease results in a significantly increased risk of mortality following burn injury compared to patients without renal disease. It is imperative to take into account comorbidities, such as renal disease, in prognosticating burn injury mortality outcomes. Further translational studies are needed to find biomarkers indicating early kidney dysfunction and potential therapeutic targets preventing further decline in renal function.

\section{Funding}

This work was supported by the National Institute of Health under award number: 5T32GM008450-23.

\section{Appendix A. Supplementary data}

Supplementary data related to this article can be found at https://doi.org/10.1016/j.amjsurg.2018.02.027.

\section{References}

1. National Kidney Foundation. About Chronic Kidney Disease; Feb 2017. https:// www.kidney.org/atoz/content/about-chronic-kidney-disease. Accessed June , 2017.

2. National Institute of Health. NIH Fact Sheets-chronic Kidney Disease and Kidney Failure; March 2013. https://report.nih.gov/nihfactsheets/ViewFactSheet.aspx? csid=34. Accessed June 22, 2017.

3. National Institute of Diabetes and Digestive and Kidney Diseases. Kidney Disease Statistics for the United States; December 2016. https://www.niddk.nih. gov/health-information/health-statistics/kidney-disease. Accessed July 22, 2017.

4. Renewal. Facts about Kidney Disease; 2017. http://life-renewal.org/wp/kidneydisease/facts-about-kidney-disease/. Accessed June 22, 2017.

5. Case J, Khan S, Khalid R, Khan A. Epidemiology of acute kidney injury in the intensive care unit. Critical Care Res Pract. 2013;2013:479730.

6. Normandin B, Yu W. Acute Kidney Failure. Healthline; Nov 2015. http://www. healthline.com/health/acute-kidney-failure\#overview1. Accessed June 22, 2017.

7. Charlson ME, Pompei P, Ales KL, Mackenzie CR. A new method of classifying prognostic comorbidity in longitudinal studies: development and validation. J Chronic Dis. 1987;40:373-383.

8. Needham DM, Scales DC, Laupacis A, Pronovost PJ. A systematic review of the Charlson Comorbidity Index using Canadian administrative databases: a perspective on risk adjustment in critical care research. J Crit Care. 2005;20: 12-19.

9. Shrier I, Platt RW. Reducing bias through directed acyclic graphs. BMC Med Res Meth. 2008;8:70.

10. Brusselaers N, Monstrey S, Colpaert K, Decruyenaere J, Blot SI, Hoste EAJ. Outcome of acute kidney in sever burns: a systemic review and meta-analysis. Intensive Care Med. 2010 June;36(6):915-925.

11. Tsalik EL, Willig LK, Rice BJ, et al. Renal systems biology of patients with systemic inflammatory response syndrome. Kidney Int. 2015;88(4):804-814. https://doi.org/10.1038/ki.2015.150.

12. Rangel-Frausto MS, Pittet D, Costigan M, Hwang T, Davis CS, Wenzel RP. The natural history of the Systemic Inflammatory Response Syndrome (SIRS): a prospective study. J Am Med Assoc. 1995;273:117-123.

13. Steinvall I, Bak Z, Sjoberg F. Acute kidney injury is common, parallels organ dysfunction or failure, and carries appreciable mortality in patients with major burns: a prospective exploratory cohort study. Crit Care. 2008;12:R124.

14. Coca SG, Bauling P, Schifftner T, et al. Contribution of acute kidney injury toward morbidity and mortality in burns: a contemporary analysis. Am J Kidney Dis. 2007;49:517-523.

15. Chrysopoulo MT, Jeschke MG, Dziewulski P, et al. Acute renal dysfunction in severely burned adults. J Trauma Injury Infect Crit Care. 1990;46:141-144.

16. Pruitt B. Protection from excessive resuscitation: pushing the pendulum back. J Trauma. 2000;49:567-573.

17. Cohen J. The immunopathogenesis of sepsis. Nature. 2002:420:885-891.

18. Al-Lamki RS, Wang J, Skepper JN, Thiru S, Pober JS, Bradley JR. Expression of tumor necrosis factor receptors in normal kidney and rejecting renal transplants. Lab Invest. 2001;81, 1503-15.

19. Majumdar A. Sepsis-induced acute kidney injury. Indian J Crit Care Med Peer Rev Off Publ Indian Soc Crit Care Med. 2010;14(1):14-21.

20. Schrier RW, Wang W. Acute renal failure and sepsis. N Engl J Med. 2004;351: 159-169.

21. Sabry A, Wafa I, El-Din AB, El-Hadidy AM, Hassan M. Early markers of renal injury in predicting outcome in thermal burn patients. Saudi J Kidney Dis Transpl. 2009 Jul;20(4):632-638.

22. Kellum JA, Levin N, Bouman C, et al. Developing a consensus classification system for acute renal failure. Curr Opin Crit Care. 2002;8:509-514. 